\title{
27. COMMISSION DES ETOILES VARIABLES
}

\author{
Report of Meetings
}

Presiding AT Both Sessions: G. H. Herbig.

SeCretary: J. D. Fernie.

During the Prague General Assembly, the Commission conducted two administrative sessions, as well as one general scientific meeting and two specialized discussions devoted to flare stars and to symbiotic objects.

\section{First business session, 23 August 1967}

Following the introduction of the Secretary and the interpreters (M. Bretz for French and German, V. Cessevič for Russian), the chairman reported on the activities of Commission 27 during the past 3 years; some of these are described in the main Commission report and will not be repeated here. It was announced that the next Variable Star Colloquium in the triennial BambergBudapest series will be held in Budapest in early September 1968. Commissions 27 and 42 have requested of the Union a grant of $\$ 500$ in support of the Colloquium. Furthermore, the Liège Astrophysical Symposium in 1969 will be devoted to pre-main sequence stellar evolution, a subject which partially falls in the domain of Commission 27.

Attention was called to the depository for unpublished photoelectric observations of variable stars, now established in the Library of the Royal Astronomical Society in London. The establishment of a second such center in Odessa, to serve the astronomers of Eastern Europe, is under consideration.

Mention was also made of the fact that, through the courtesy of the National Geographic Society and the Mt. Wilson and Palomar Observatories, the rules for publication of Palomar Atlas copies had been eased, in cases where the reproductions are used for purposes of variable star identification. The details of this arrangement were described in a circular sent to members of the Commission in March 1965.

The President then turned to questions having to do with the General Catalogue of Variable Stars. He read extracts from a letter from Dr B. Kukarkin, which described this work and some of the problems of the Moscow bureau. The work there began in 1940, and was taken up again in 1946 following the interruption caused by the war and in response to a proposal from the Executive Committee of the Union that it accept the task of naming new variables, and publishing the necessary catalogues. The first edition of the GCVS (listing 10912 variables) appeared in 1948, and in the following ten years the bureau issued 9 Supplements, published (in Leiden) 5 lists of new designations, and in 1950 issued the Catalogue of Stars Suspected of Variability. The second edition, in two volumes and containing even more complete information than the first, appeared in 1958 . It lists 14708 stars. Two Supplements have been published, with designations for 4083 new variables and more precise data on 1647 others. (A copy of the Second Supplement was presented by Cessevič during the meeting). A table of galactic coordinates of variable stars, compiled by L. Plaut, and a Second Catalogue of Stars Suspected of Variability with information on 3907 stars have been published.

The third edition of the GCVS is now nearing completion. It will contain information on $18791+N$ variables, where $N$ is several hundred, representing the last group of new variables that will be assigned names in the fall of 1967.

The bureau's work of collecting and analyzing variable star data has been progressively improved, some processes having been automatized. Despite this, the volume of work has greatly increased. The staff of the bureau must still read and analyse the literature and letters; this cannot be done by machines. On account of the increasing complexity of variable star work, highly qualified 
personnel are essential for this task. The result is that the astronomical staff have become slaves to this flood of information, and have little time left for their own scientific work. It is evident that this small group, which has not been enlarged since 1946, will soon be unable to cope with the task, despite the unselfish help given them by their colleagues of the Sternberg Astronomical Institute.

Therefore Commission 27 has been asked by Kukarkin to support the formal request made to the Union by the Astronomical Council, USSR Academy of Sciences, for financial support. A grant of $\$ 5000$ was requested for extraordinary assistance toward the preparation of the third edition of the GCVS, and an annual subvention of $\$ 500$ for continuing technical support. (Kukarkin mentioned, incidentally, that the bureau has to spend much time on checking and correcting star coordinates that have been determined carelessly.)

In addition to these activities, the Variable Star Commission of the Astronomical Council is responsible for publishing the bulletin Variable Stars (Peremennye Zvezdy) and the Astronomical Circulars, which impose an additional financial burden. Kukarkin asked for a statement by Commission 27 whether it would favor the transformation of Variable Stars into a subscription journal. This would make it possible, if enough subscriptions were obtained, not only to release the staff of the bureau from this technical labor but to issue the journal more regularly, to expand its format, and to publish papers in languages other than Russian.

Finally, Kukarkin expressed on behalf of himself and his staff the deepest gratitude to the world-wide community of variable star investigators for their friendly help.

The Commission formally endorsed the principle that Variable Stars be placed on a subscription basis. The President commented that the financial position of the Union was such that it was very unlikely that support in these amounts could be expected. The President made the following interim suggestion: the Organizing Committee of Commission 27 wishes to recommend, and asks approval of the Commission, that in the future not only Variable Stars be placed on a subscription basis, but that the GCVS and its Supplements be sold instead of being distributed without charge, as is the practice at present. If this were done, presumably the amount of the grant requested for assistance to the Moscow bureau could be decreased. The Commission could consider the matter again at its second business meeting, by which time the reaction of the Soviet delegation to this counter proposal would be known. The Commission unanimously endorsed this suggestion.

A proposal by W.J. Miller was presented, to the effect that the Moscow bureau modify its present procedure of assigning variable star designations. In essence, present practice is that new variables are not named until just before a new Supplement is completed, at which time the designations are assigned in order of right ascension. Miller's proposals were (1) that the assignment of names be performed more frequently, almost as soon as the reality of the variation is accepted, without waiting for a final ordering of stars according to right ascension; and (2) that these new designations be immediately available on inquiry. In the discussion which followed, the possibility of publishing frequent Benennungsiste was raised. Cessevič indicated that it would be impossible to publish full details on a short time scale, but that publication of simple lists of new names might be feasible. It was mentioned that use might be made of the Information Bulletin of Commission 27 for this purpose. The President finally proposed that the details be left to the consideration of Kukarkin and his associates, and that the Commission simply convey its opinion to them that the assignment of designations should be made more frequently, and that the possibility of reasonably rapid announcement of these designations be examined. This proposal carried unanimously.

In 1961, a Working Group of Commission 27 under the chairmanship of Dr A. Wesselink was appointed to recommend a system of nomenclature for variable stars in extragalactic systems. Since the most urgent problem exists in the case of the Magellanic Clouds, the Group has given its main attention to that question. Wesselink presented its final recommendations to the Commission: (1) An area surrounding each cloud be defined by the following corner points (for the equinox 1875.0 , in order to be compatible with the official constellation boundaries), connected by meridians or small circles: 


$\begin{array}{ccc}\text { Small Cloud: } & & \text { Large Cloud: } \\ 0^{\mathrm{h}} 00^{\mathrm{m}},-69^{\circ} 00^{\prime} & 4^{\mathrm{h}} 35^{\mathrm{m}},-64^{\circ} 00^{\prime} \\ 140,-6900 & 635,-6400 \\ 140,-7700 & 635,-7400 \\ 000,-7700 & 435,-7400\end{array}$

(2) Within these two areas, all variable stars will be assigned designations not according to constellation but on the new system, regardless of whether they are physical members of the Clouds or not. Non-variable stars in these areas will not be affected in any way. (3) A new system of designations will be devised which will contain an indication whether the star falls in the LMC or SMC area.

In other words, all stars in these two areas will be removed from future $G C V S$, and will appear only in a special catalogue of Magell anic Cloud variables. Some special indication may be given to stars that are known to be foreground objects. This mixing of Cloud members and non-members is an annoyance, but it is conventionally accepted in galactic and globular clusters and in some extragalactic systems. After some discussion, the President proposed (a) that the Commission accept the recommendations of the Working Group; (b) that the criteria for the acceptance of a new variable into the new system be fully as stringent as those of the GCVS; (c) that technical details, such as the designations themselves, be left to a new Working Group which the Organizing Committee would appoint for the task of actually compiling the new Catalogue. He would report on some administrative details in the second business session. The Commission endorsed these proposals unanimously.

Dr Cessevič exhibited a copy of a limited photographic edition of finding charts for some 5000 variable stars that had been prepared by the Odessa Observatory. Copies of this Atlas had been deposited at 20 observatories active in variable star work.

In conclusion, Dr Oosterhoff complimented Drs Herbig, Feast, and Sawyer-Hogg on the Report of Commission 27.

\section{Second business session, 29 August 1967}

The President announced that a small bureau will be established at the Mt. Stromlo Observatory, under the direction of Dr S. Gascoigne, for the preparation of a Catalogue of Magelianic Cloud variables following the recommendations of the Wesselink Group (which had now been discharged, with thanks for their labors). To assist and advise Dr. Gascoigne, a new Working Group had been established under his chairmanship, composed of representatives of the major centers of work on Cloud Variables: S. Gaposchkin (Harvard), B. Kukarkin (Moscow), J. Landi Dessy (Córdoba), P. Th. Oosterhoff (Leiden), A. Thackeray (Pretoria), R. Woolley (Herstmonceux).

It was reported that as a result of the Commission's proposal at the earlier session, the Soviet request for financial support for the work of the Moscow bureau had been modified. The President explained that it would be desirable to identify for the Executive Committee the precise terms on which the Commission might agree to support this request, and so he moved the following resolution:

Commission 27 recommends that, in view of the important services of cataloguing and systematizing variable star data that are performed by the Variable Star bureau of the Astronomical Council, USSR Academy of Sciences, the IAU give partial financial support to this work in the amount of a single grant of $\$ 2000$, plus an annual subvention in the amount of $\$ 500$. We recommend these funds specifically to support (1) the reorganization of the work of the bureau, and (2) continued free distribution of publications to members of Commissions 27 and 42, with the publications being sold to all other individuals and institutions.

The Commission unanimously endorsed this resolution.

Dr Detre discussed the need for a concentrated observational effort to determine secondary periods for RR Lyrae stars in a few selected globular clusters, particularly M3, M5, M15. About 3000 plates should be obtained of these three clusters over a period of a few months, which implies an observatory located in an excellent climate. He pointed out also the importance for the same purpose of old plate material, especially that in the files of the Harvard and Mt. Wilson Obser- 
vatories. After some discussion of the accessibility of those plates to outside investigators, the Commission endorsed a resolution supporting and encouraging such a intensive observational program on globular cluster RR Lyrae stars.

The President described the identification atlas of old novae that was being prepared by $\mathrm{Dr}$ Ch. Bertaud. Several sample charts from this atlas were displayed. This enterprise is especially important because of great interest being shown in these stars at the present time. The Commission voted a resolution expressing strong support and encouragement for Dr Bertaud's work. Attention was also called to a list prepared by Dr Bertaud of possibly recurrent novae which deserve continuing attention. Copies were available at the meeting for those interested, and a condensed version appears as an appendix to this report.

The subject of possible new activities for amateur associations was introduced by the President. He pointed out that Commission 27 has no authority or intention of interfering with the activities of volunteer observers. For amateurs, variable star work is a hobby like collecting stamps, and professional astronomers cannot tell amateurs what to do with their spare time. The only reason for bringing the subject up is this: the major activity of the associations is following Mira variables. These programs were initiated over a half-century ago, and met a real need of the astronomy of that era. Without minimizing the usefulness of such observations even today, one must admit that astronomy has since become a much broader subject. Are there some other programs suitable for amateur observers that might be equally profitable in the light of today's science? Almost every astronomer could suggest a few possibilities; the following is a somewhat biased list:

(1) A more thorough patrol of recurrent novae, both known and suspected (see the Bertaud list for candidates).

(2) A thorough and systematic study of the brighter flare stars, to detect both individual flares and prolonged periods of high activity.

(3) Accurate timing of minima of short-period binaries of special interest: for example, U Gem, VSge, RWTri.

(4) Systematic observation of T Tauri stars that are known to have occasional nova-like outbursts: VY'Tau, UZTau, EXLup.

(5) A number of interesting irregular variables are apparently not being followed at all: WW Vul, BFOri, XY Per A + B, BNOri.

Feast stressed the point that continuing work by amateurs on long-period variables was very important and useful, but that an improvement in photometric sequences that they used was also very necessary. Mrs. Mayall agreed, and said that the amateur groups would be glad to add other stars to their programs, if only good sequences could be provided. She indicated that perhaps those amateurs who have photoelectric photometers might be able to supply such data.

Mrs Mayall also reported that it was planned eventually to record all 2.5 million AAVSO observations on punched cards, and a beginning has been made. She welcomed advice on the best format for publication (10-day means, plots, or individual observations), and whether it was desirable to combine these results with those of other amateur organizations. The President suggested that interested persons discuss these questions directly with Mrs Mayall.

Hoffmeister presented a short report on the comprehensive program carried out at Sonneberg to investigate the statistical properties of variable stars, and their light curves. The significance of such work was emphasized by the Commission in a resolution:

Commission 27 stresses the great importance of statistical studies of the types and distribution of variable stars for investigations of the structure and evolution of the galaxy.

The President noted that the late Dr H. Schneller had left unfinished in Potsdam the manuscript of a new edition of the Geschichte und Literatur. Such bibliographical monographs were extremely useful to variable star investigators, and everyone would surely agree on the desirability of completing and publishing this new edition. It was unanimously resolved that:

Commission 27 strongly supports and encourages the preparation of a new edition of the $\mathrm{Ge}$ schichte und Literatur der Veränderlichen Sterne.

The Commission was informed of the discussion of flare star problems that had taken place a 
few days previously in a small meeting of specialists. L. Geršberg stressed the importance of continuous photoelectric observations of flare stars. A continuous 24-hour coverage from observatories all around the earth is desirable on some special occasions, as had been organized in the past to support radio observations of flare stars. Although the organization of such cooperative efforts is in the province of the IAU, Commission 27 is too large a body to direct such a program. It was also pointed out that there was a real need for more systematic exchange of data, preprints, information on programs, etc. For these reasons, the President appointed a Working Group on Flare Stars to discuss these problems in detail, and organize cooperative programs as they saw fit. The following astronomers were designated as the nucleus of the Working Group: A. D. Andrews, P. F. Cugainov, L. Geršberg, V. Oskanian. They were empowered to select their own chairman (Dr Cugainov was subsequently elected) and to co-opt any other members that could assist in their task, with special attention being given to radio astronomers. The Commission supported this work through the following resolution:

Commission 27 emphasizes the importance of cooperative studies of flare stars, especially those based on simultaneous photoelectric, spectroscopic and radio observations during periods of 24 hours and longer.

Miss Hoffleit reported to the Commission an inquiry she had received (from scientists outside the variable star field) regarding the possibility of compiling a catalogue of all existing astronomical direct photographs. She felt that this would be an impractically large task, but asked for opinion whether a list of observatories which have such material might be compiled, together with a general description of the nature and time coverage of the photographs. Following some discussion, the question was deferred until a more detailed and specific proposal could be put before the Commission.

Hill discussed briefly the significance of the hot helium stars $+10^{\circ} 2179$, HD 124448, HD $168476, \ldots$ which seem to have chemical compositions much like the $\mathrm{R} \mathrm{CrB}$ variables. He pointed out the importance of a careful photometric study of the hot He stars to find if any are indeed variable.

$P$. Kulikovsky called attention to a list of double $\mathrm{T}$ Tauri stars published in Information Circular of Commission 26, Nos. 31 and 32. He urged special attention be given to these stars.

In conclusion, the President presented the slate of new officers of Commission 27 that was proposed by the present Organizing Committee, in consultation with the General Secretary:

President: L. Detre.

Vice-President: O. J. Eggen.

Organizing Committee: A. Bojarčuk, R. Kippenhahn, K. Kwee, G. Odgers, G. Herbig.

The Commission indicated its approval of this new panel of officers. The meeting then adjourned.

\section{Appendix}

POSTNOVAE TO BE EXAMINED FOR RECURRENCE

\section{Ch. Bertaud}

All the known recurrent novae have amplitudes less than 9 magnitudes (TPyx: $\Delta m=6$ m 6 ; RSOph: $7^{m} .2$; TCrB: $8^{m} \cdot 0$; WZSge: $8^{m} \cdot 1$ ) except possibly VYAqr, for which the amplitude is not known. The old novae in the following list conform to this $\Delta m<9^{m}$ limit, and thus deserve particular attention.

HR Lyr $1919\left(\Delta m=8^{m} .5\right)$. This amplitude corresponds to a minimum magnitude of $15 \cdot 0$, but the star is photographically very active. Between 1947 and 1952, it varied between $14^{m} .25$ and $15^{\mathrm{m}} .30$ (L. Rosino and A. Betti, Pubbl. Bologna 5, No. 21, 1952). One Meudon plate of 1962 Aug. 24 gave $15^{m} .0$.

IM Nor $1920\left(7^{m} .5:\right)$. The identification of the prenova at $16^{m} .5$ is uncertain (S.I. Bailey, Harv. Bull. 734,1920 ). There are only a few observations of this star during the month-long maximum at 9.0 . 
V1016 Sgr $1899\left(6^{m} .5\right)$. The maximum may not have been observed; an extrapolated maximum of $7 . m .0$ corresponds to an amplitude of $7 . \mathrm{m} \cdot 9$.

HSSgr $1900\left(6^{m} .5:\right)$. The probable magnitude of the prenova according to Miss Woods is $16^{m} .5$, and the extrapolated maximum according to McLaughlin is $10^{\mathrm{m}} .0$.

V999Sgr $1910\left(8^{m} .4\right)$. The magnitude 26 years after the outburst was $16^{m} \cdot 6$ (H.H. Swope, Harv. Bull. $913,11,1942)$; the maximum magnitude is assumed to be 8.2 .

V1017Sgr $1919(7.0$ ). The star now is slowly variable near mag. 14 (M.W. Mayall, J. R. astr. Soc. Can., 57, 127, 1963). The small amplitude in 1919, and the existence of a minor outburst in 1901 (D. B. McLaughlin, Publ. astr. Soc. Pacific, 58, 46, 1946), justifies continued surveillance.

FNSgr 1925 (5 .0 ). The spectrum resembles that of an old nova (G.H. Herbig, Publ. astr. Soc. Pacific, 62, 215, 1950).

FM Sgr 1926 (8. $5:)$. The extrapolated maximum magnitude is 8.0 , while the prenova seemed to be of mag. 16 or 17 . The amplitude is therefore uncertain.

V441 Sgr $1930\left(7^{m} .3\right)$. The amplitude given here is obtained from a maximum magnitude of 8.7 , but it is probable that the real maximum magnitude was about 8.0 ; the amplitude was on this basis about 8 mag.

EUSct $1949(8 \mathrm{~m})$. The minimum magnitude of 16.8 follows from observations of the prenova between 1918 and 1949 (M. Harwood, Leiden Ann., 21, 387, 1962).

XSer $1903\left(6^{m} .0\right)$. The maximum of $9^{m} .0$ was attained in 1903. At minimum the star oscillates around $15^{m}$ with a range of 2 mag., in a fashion resembling ZAnd. There is a resemblance to the quasiperiodic oscillations of RR Tel before its outburst (C. Payne-Gaposchkin, The Galactic Novae, p. 192, 1957). 